\title{
Meta-Analysis of the Efficacy and Safety of Finerenone in Diabetic Kidney Disease
}

\author{
Yaning Zheng ${ }^{\mathrm{a}}$ Sheng Ma ${ }^{\mathrm{b}}$ Qiaomu Huang ${ }^{\mathrm{a}}$ Yu Fang ${ }^{\mathrm{a}}$ Hongjin Tan $^{\mathrm{a}}$ \\ Yong Chen ${ }^{\mathrm{a}}$ Cairong $\mathrm{Li}^{\mathrm{a}, \mathrm{c}}$ \\ aDepartment of Nephrology, Xianning Central Hospital, First Affiliated Hospital of Hubei University of Science and

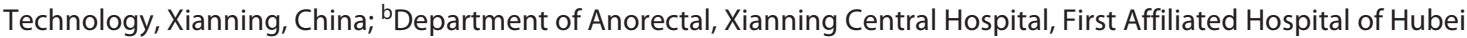 \\ University of Science and Technology, Xianning, China; 'Xianning Medical College, Hubei University of Science and \\ Technology, Xianning, China
}

\section{Keywords}

Finerenone (BAY 94-8862) · Safety · Diabetic kidney

disease $\cdot$ Efficacy $\cdot$ Renal function

\begin{abstract}
Background: The phase III clinical trial of the nonsteroidal mineralocorticoid receptor antagonist finerenone (BAY 948862 ) has been completed, aiming to investigate renal and cardiovascular outcomes in type 2 diabetes (T2D) with chronic kidney disease (CKD). However, the efficacy and safety of finerenone in renal function remain controversial. The purpose of this study was to explore the efficacy and safety of finerenone in treating the patients with diabetic kidney disease (DKD). Methods: Databases of PubMed, Cochrane Library, Embase, and Web of Science were searched for randomized controlled trials (RCTs) on patients with DKD receiving finerenone treatment from inception to September 2021. Data including patient characteristics and interested outcomes were extracted, and the dichotomous data and continuous variables were evaluated using risk ratio (RR) with $95 \%$ confidence intervals (Cls) and mean differences (MD) with $95 \% \mathrm{Cls}$, respectively. Results: A total of 4 RCTs involving 13,945 patients were included in this meta-analysis. Analysis results demonstrated that patients receiving finerenone showed a significant decrease in changing urinary
\end{abstract}

albumin-to-creatinine ratio (UACR) from baseline (MD: -0.30 ; $\left.95 \% \mathrm{Cl}[-0.33,-0.27], p=0.46, I^{2}=0 \%\right)(p<0.05)$. The number of patients with $\geq 40 \%$ reduction in estimated glomerular filtration rate (eGFR) from baseline in the finerenone group was significantly smaller than that in the placebo group (RR: $\left.0.85 ; 95 \% \mathrm{Cl}[0.78,0.93], p=0.60, l^{2}=0 \%\right)(p<0.05)$. No difference was found in adverse events between the finerenone and placebo groups (RR: 1.00; 95\% Cl [0.98, 1.01], $p=$ $\left.0.94,1^{2}=0 \%\right)(p=0.65)$. The incidence of hyperkalemia was higher in the finerenone group than that in the placebo group (RR: $\left.2.03 ; 95 \% \mathrm{Cl}[1.83,2.26], p=0.95, R^{2}=0 \%\right)(p<$ $0.05)$. Conclusion: Finerenone contributes to the reduction of UACR and can ameliorate the deterioration of renal function in patients with T2D and CKD. The higher risk of hyperkalemia was found in the finerenone group compared with placebo; however, there was no difference in the risk of overall adverse events.

(c) 2022 The Author(s).

Published by S. Karger AG, Basel

\section{Introduction}

Chronic kidney disease, defined as abnormal renal function for more than 3 months, has affected large part of population and resulted in a heavy burden in treatment cost around the world $[1,2]$. As one of the traditional risk factors
Karger@karger.com www.karger.com/kbr

Karger $\stackrel{\text { ' }}{5}$

GOPEN ACCESS
(C) 2022 The Author(s)

Published by S. Karger AG, Basel

This is an Open Access article licensed under the Creative Commons Attribution-NonCommercial-4.0 International License (CC BY-NC) (http://www.karger.com/Services/OpenAccessLicense), applicable to the online version of the article only. Usage and distribution for commercial purposes requires written permission.
Correspondence to:

Sheng Ma,124765760@qq.com

Cairong Li, xnlcr@163.com 
for $\mathrm{CKD}$, diabetes mellitus was a prevalent disease in the world [3]. The incidence of diabetes mellitus around the world was 9.3\% (463 million people) in 2019 as estimated and will rise to $10.2 \%$ (578 million people) by 2030 and $10.9 \%$ (700 million people) by 2045 [4]. Approximately $30-$ $40 \%$ of T2D patients will develop into DKD, with the typical clinical features of persistent albuminuria or eGFR $<60$ $\mathrm{mL} / \mathrm{min} / 1.73 \mathrm{~m}^{2}$ for more than 3 months [5]. DKD with various renal pathological changes (such as extracellular matrix deposition, thickening of glomerular basement membrane, tubule atrophy, and finally, glomerulosclerosis and tubulointerstitial fibrosis) is the leading cause of endstage kidney disease in the developed world [6]. With a 3 -fold higher risk of cardiovascular death than those with T2D alone [7], patients suffering from DKD may eventually require chronic renal replacement therapy as renal impairment progresses. However, treatments to slow the progression of DKD are limited, and current therapies are wildly used as a cornerstone, including angiotensin-converting enzyme inhibitors, angiotensin II receptor blockers, and sodium-glucose cotransporter 2 inhibitors [8-11].

Finerenone (BAY 94-8862), a novel nonsteroidal mineralocorticoid receptor antagonist, shows a higher selectivity toward the mineralocorticoid receptor and low affinity for androgen, glucocorticoid, and progesterone receptors in vitro $[12,13]$. As the third generation of the nonsteroidal mineralocorticoid receptor antagonist drug, finerenone demonstrates better protective effects on cardiorenal function compared with spironolactone or eplerenone due to the balanced tissue distribution into the heart and kidney [14-18], as well as a lower incidence of adverse events such as hyperkalemia [14]. In recent years, various research studies were conducted on the clinical effectiveness and safety of finerenone in cardiovascular and kidney diseases $[19,20]$. Owing to a lack of relevant reviews reporting the efficacy and safety of finerenone on the renal function of DKD patients, this meta-analysis of randomized, placebo-controlled trials was conducted to evaluate the efficacy and safety of oral finerenone as a treatment for DKD.

\section{Methods}

\section{Search Strategy}

We searched PubMed, the Cochrane Library, Embase, and Web of Science for eligible studies published in English from inception to September 2021, with the search terms "Chronic kidney disease" and "Finerenone." The detailed search strategy is available in online supplementary Table S1 (for all online suppl. material, see www.karger.com/doi/10.1159/000521908).
Inclusion and Exclusion Criteria

Studies meeting these criteria were considered eligible: (1) patients (age $\geq 18$ years) with T2D and chronic kidney disease (UACR $\geq 30 \mathrm{mg} / \mathrm{g}$ and $\mathrm{eGFR} \leq 90 \mathrm{~mL} / \mathrm{min} / 1.73 \mathrm{~m}^{2}$ ) who previously received $\geq 4$ weeks of treatment of angiotensin-converting enzyme inhibitors or angiotensin II receptor blockers or both; (2) oral finerenone as an intervention at any doses; (3) placebo applied in the control group; (4) at least one interested outcome was reported; (5) RCTs published in English. The exclusion criteria were as follows: (1) patients with a serum potassium concentration $\geq 4.8$ $\mathrm{mmol} / \mathrm{L}$; (2) patients receiving renal replacement therapy; (3) patients with glycosylated hemoglobin $>12 \%$; (4) animal experiments; (5) meta-analysis, review, case report, conference, and letter.

\section{Data Extraction}

Two reviewers independently extracted data from the included studies, and any disagreements were resolved through consultation with a third reviewer to reach a consensus. The extracted data included the first author, publication time, gender, country, drug dose, intervention time, sponsorship source, conflicts of interest, outcomes, and results. The outcomes included the changing UACR from baseline, the incidence of $\mathrm{a} \geq 40 \%$ reduction in eGFR from baseline, serum potassium $\geq 5.6 \mathrm{mmol} / \mathrm{L}$, and adverse events. Risk of bias was explored according to the Cochrane Handbook for Systematic Reviews of Interventions (the detailed list was as follows: selection bias [random sequence generation, allocation concealment], performance bias [blinding of participants and personnel], detection bias [blinding of outcome assessment], attrition bias [incomplete outcome], reporting bias [selective reporting], and other bias [such as conflicts of interest]) [21].

\section{Data Synthesis and Analysis}

The dichotomous data of outcomes were presented as a risk ratio (RR) with $95 \%$ confidence intervals (CIs), while the continuous variables of outcomes (changing from baseline to certain follow-up time) were reported as mean differences (MD) with $95 \%$ CIs. The fixed-effects model was adopted for meta-analysis when no significant heterogeneity was observed $\left(I^{2}<50 \%, p \geq 0.1\right)$, otherwise the random-effects model was used. Sensitivity analysis was conducted to find the heterogeneity source by sequentially excluding every single study. Statistical analysis was conducted with RevMan 5.3, and $p<0.05$ was considered as statistically significant.

\section{Results}

\section{Characteristics of the Included Studies}

The detailed flow chart of study selection is presented in Figure 1. A total of 197 articles were screened by title and abstract after removing duplicates, and 6 of which were further reviewed in full text [16, 22-25]. Four RCTs were finally included in this meta-analysis $[16,22,23,26]$. Two studies compared daily dose of 2.5-20 mg finerenone with placebo $[16,22]$, and the other two studies compared daily dose of 10 or $20 \mathrm{mg}$ finerenone with the placebo $[23,26]$. The detailed characteristics of the stud- 
Fig. 1. Flow diagram and description of study selection process.

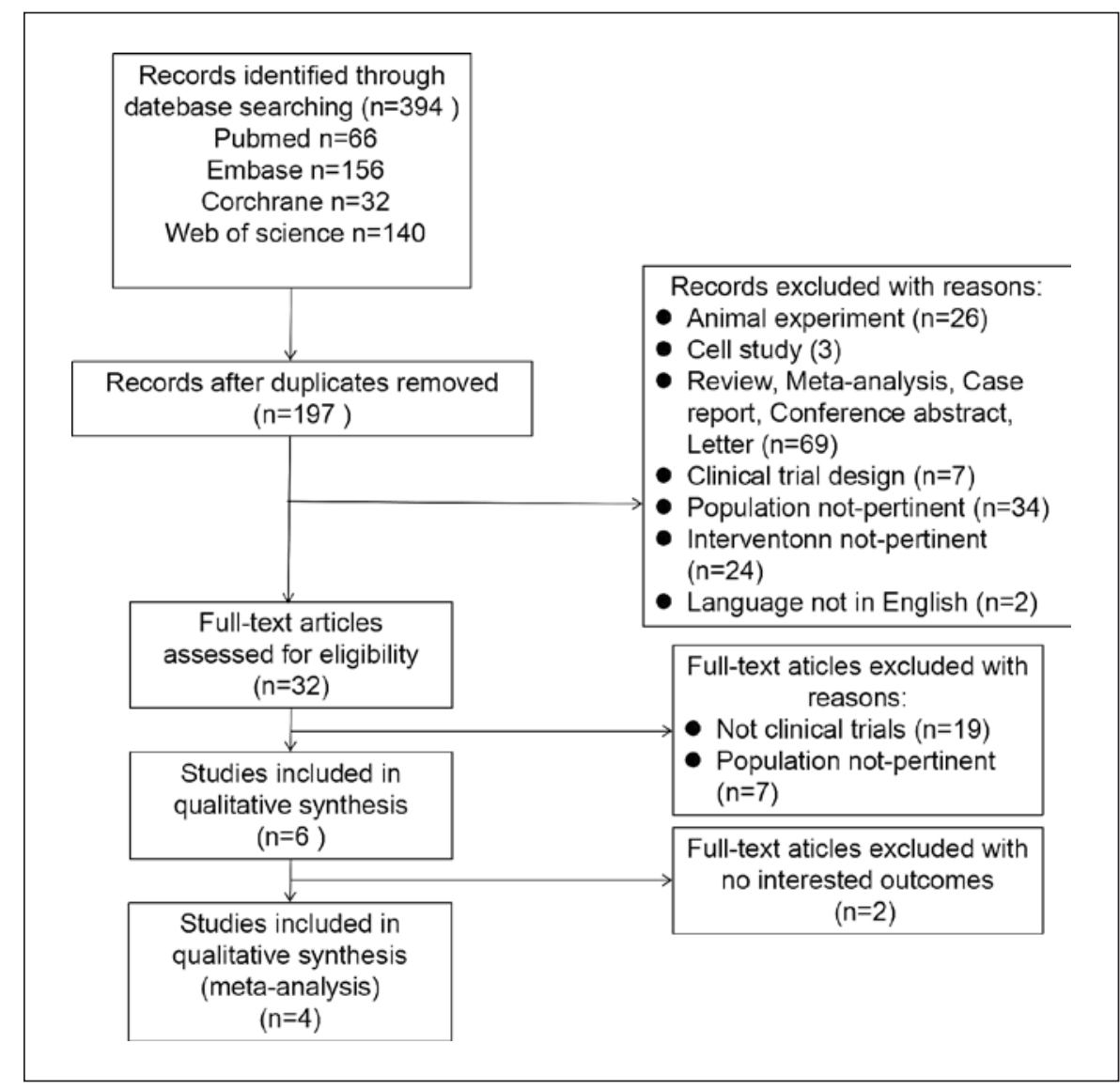

ies are presented in Table 1. The risk of bias was evaluated by using RevMan 5.3 for the overall quality of the included articles (Fig. 2). The missing data of UACR resulted in high risk of bias of incomplete outcome data in Bakris 2020 [23]. All the four studies were sponsored by the same company that developed finerenone, which might lead to potential financial conflicts of interest.

\section{Primary Outcomes: UACR and eGFR}

With the extracted data of the changing UACR from baseline to follow-up time, we pooled the results of finerenone at different doses in the studies by Bakris et al. [16] and Katayama et al. [22], as the data of the finerenone group. The fixed-effects model was applied because there was no significant heterogeneity $\left(p=0.46, I^{2}=0 \%\right)$. The pooled analysis of four RCTs revealed a significant decrease of changing UACR from baseline in the finerenone group compared to the placebo group (MD: -0.30 ; $95 \%$ CI $\left.[-0.33,-0.27], p=0.46, I^{2}=0 \%\right)(p<0.05)$ (Fig. 3a). The data of UACR in the four RCTs were stable as proved by sensitivity analysis (Fig. 4).

Meta-Analysis of the Efficacy and Safety of Finerenone in DKD
The outcome of eGFR was described as the incidence of a $\geq 40 \%$ decrease in eGFR from baseline. The pooled data of four RCTs indicated that patients with T2D and CKD in the finerenone group had a lower risk of $\geq 40 \%$ decrease in eGFR from baseline compared with the placebo group (RR: $0.85 ; 95 \%$ CI $[0.78,0.93], p=0.60, I^{2}=$ $0 \%)(p<0.05)$ (Fig. 3b).

\section{Secondary Outcome: Adverse Events}

There was no significant heterogeneity in the outcome of adverse events $\left(p=0.94, I^{2}=0 \%\right)$, and thus the fixedeffects model was employed. There was no difference between the finerenone and placebo groups in adverse events reported in the four RCTs (RR: $1.00 ; 95 \%$ CI [0.98, 1.01], $\left.p=0.94, I^{2}=0 \%\right)(p=0.65)$ (Fig. 5a). The pooled results of four RCTs showed that adverse events related to hyperkalemia were more common in the finerenone group than in the placebo group (RR: $2.03 ; 95 \%$ CI [1.83, 2.26], $\left.p=0.95, I^{2}=0 \%\right)(p<0.05)$ (Fig. 5b). Sensitivity analysis demonstrated the data of adverse events were stable (Fig. 4). 


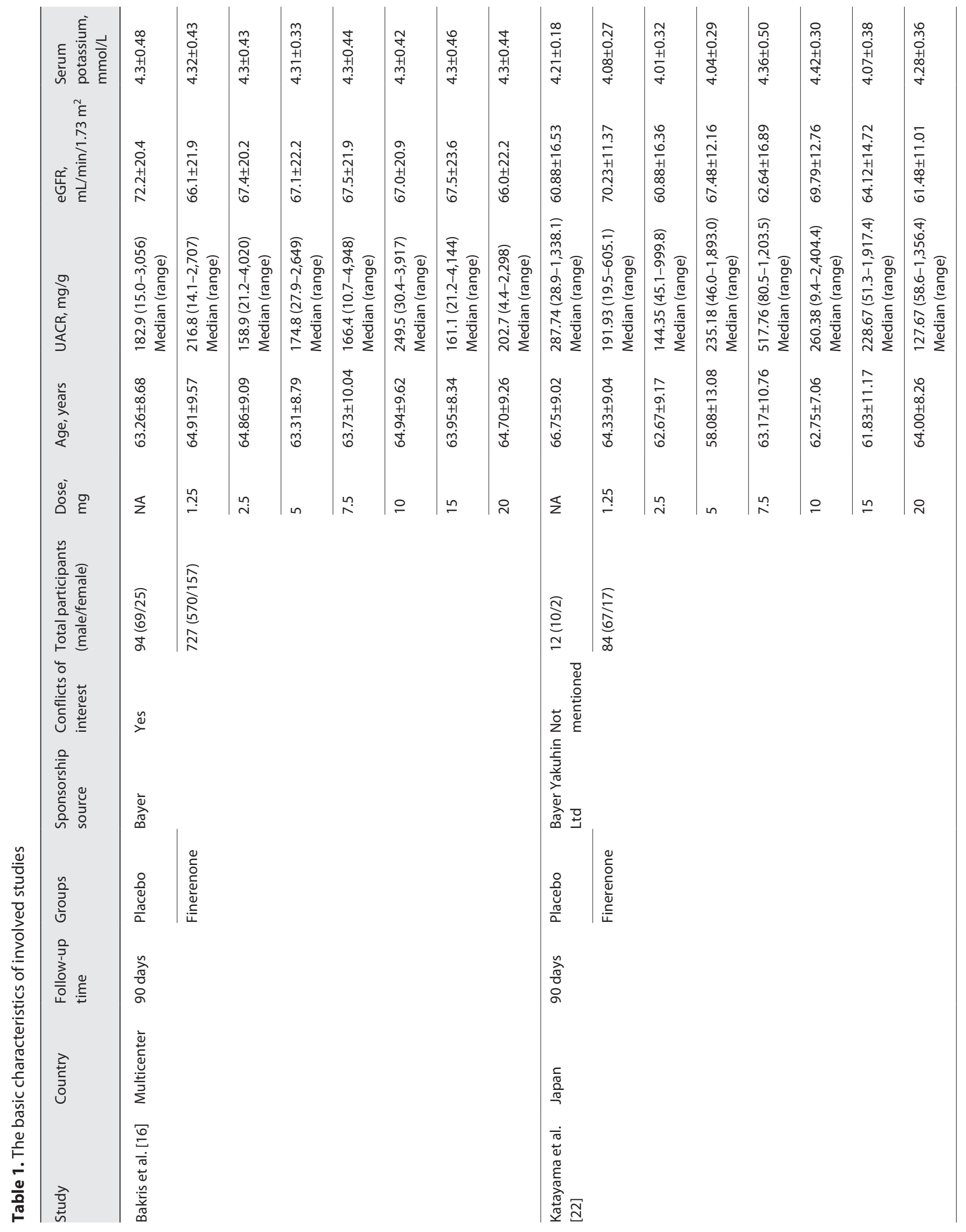




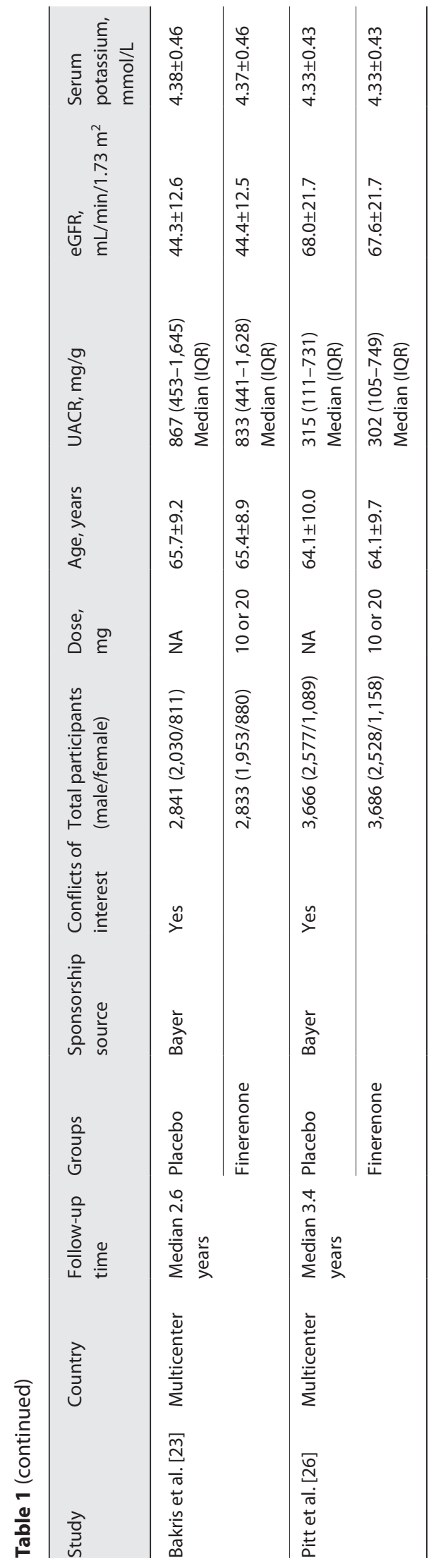

Meta-Analysis of the Efficacy and Safety of Finerenone in DKD

\section{Discussion}

The phase III clinical trial for finerenone (BAY 948862 ), a new generation of the nonsteroidal mineralocorticoid antagonist, was finished in 2021 for investigation of renal and CV outcomes in T2D patients with CKD. Despite the lack of trials on finerenone in treating DKD, our study included four RCTs (ARTS-DN, ARTS-DN Japanese, FIDELIO-DKD, and FIGARO-DKD) involving 13,945 patients. The relationship between albuminuria and deteriorating renal function in diabetic nephropathy has been confirmed [27-29]. In this meta-analysis, patients treated with finerenone showed a significant decrease in changing UACR from baseline, which indicated that finerenone could slow the progression of CKD by reducing albuminuria in diabetes. Meanwhile, the ARTSDN study showed that finerenone induced a dose-dependent reduction in UACR [16]. Although the risk of eGFR reduction in the finerenone group was lower than that in the placebo group, an interesting phenomenon was observed in our research. The change of eGFR in the finerenone group was similar to that in the placebo group in the 90-day observation and in FIGARO-DKD (with a mean eGFR of 66 and $68 \mathrm{~mL} / \mathrm{min} / 1.73 \mathrm{~m}^{2}$, respectively) [16, 22, 26], but patients treated with finerenone had a lower risk of eGFR reduction compared with those receiving placebo in FIDELIO-DKD (with a mean eGFR of $44 \mathrm{~mL}$ / $\min / 1.73 \mathrm{~m}^{2}$ ) [23], suggesting that treatment of finerenone can delay the deterioration of renal function in T2D patients with moderate chronic kidney disease.

Previous studies have reported the renoprotection of finerenone in patients with chronic heart failure and mild to moderate chronic kidney disease $[14,30,31]$ through its anti-inflammatory and antifibrotic effects $[15,17,18$, 32]. Kolkhof et al. [15] illustrated that the anti-inflammatory and antifibrotic marker, osteopontin, was dose-dependently decreased by finerenone in cardiac tissue in the deoxycorticosterone acetate-/salt-treated rats, and proteinuria was also dose-dependently reduced. Another study showed that finerenone ameliorated albuminuria and normalized endothelial dysfunction in the aorta of Munich Wistar Frömter rats by increasing endothelial NO availability [33]. Recently, the possible potential mechanisms of finerenone in cardiovascular and kidney diseases are still under study.

In spite of no difference between finerenone and placebo in adverse events, the risk of hyperkalemia was higher in the finerenone group than that in the placebo group. The incidence of hyperkalemia was similar in the finerenone and placebo groups during the 90-day 


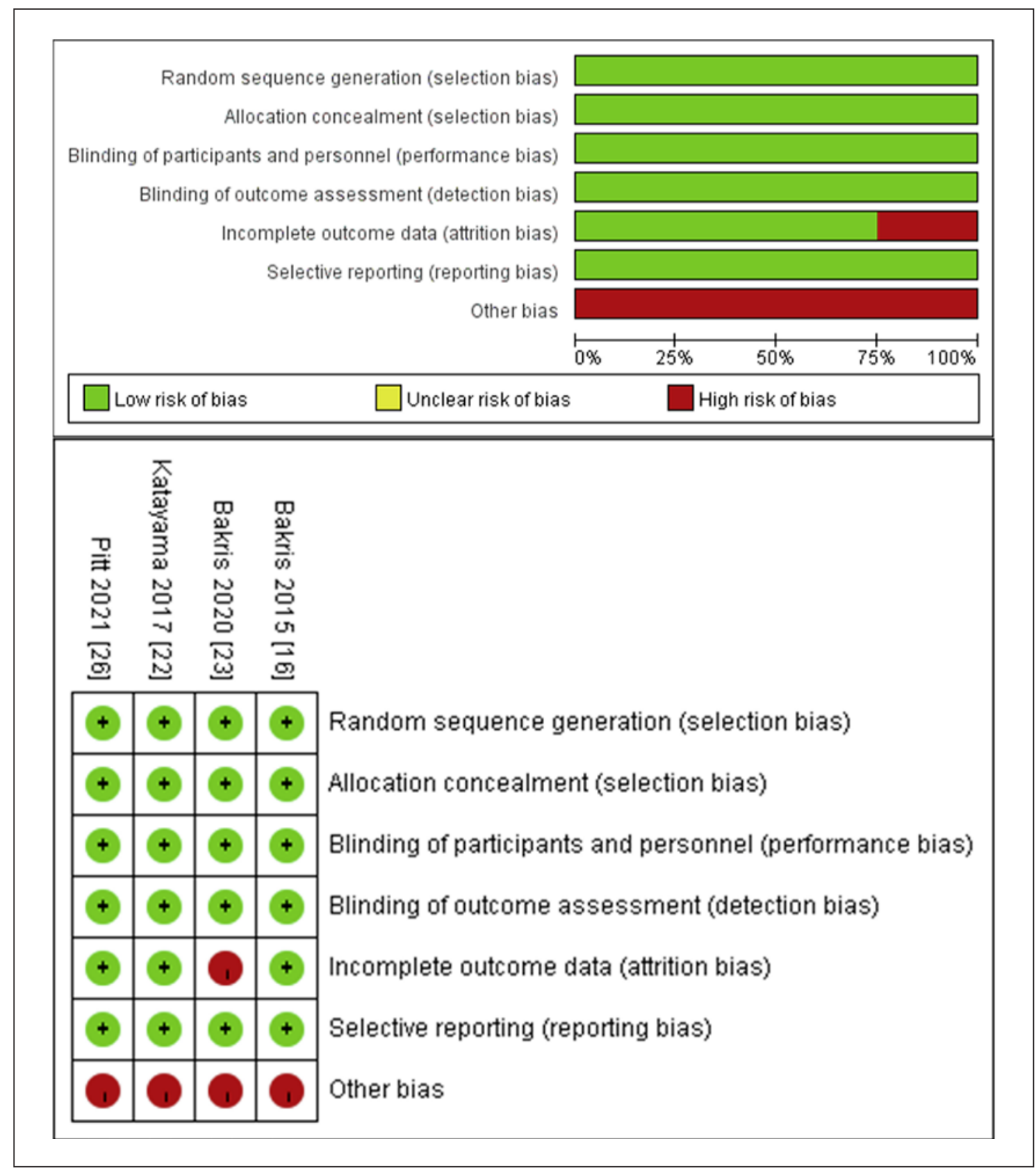

Fig. 2. Chart and summary for risk of bias.

observation, but higher in the finerenone group in the long-term observation. We considered that participants in FIDELIO-DKD and FIGARO-DKD received a higher dose (10 or $20 \mathrm{mg} /$ day) of finerenone, which could induce more reabsorption of potassium in the connecting tubules and cortical collecting ducts in the kidney [34-36]. Even so, the incidence of hyperkalemia caused by finerenone $(10 \mathrm{mg} /$ day $)$ was lower than that by spironolactone or eplerenone (25-50 mg/day) [19]. Similarly, a dose of $10 \mathrm{mg}$ /day finerenone also provided better protective effects on the cardiovascular system in comparison with spironolactone or eplerenone at a dose of 25-50 mg/day [37]. Despite the higher incidence of hyperkalemia in the finerenone group reported by FIDELIO-DKD and FIGARO-DKD, no fatal hyperkalemia-related event occurred. Another finding 


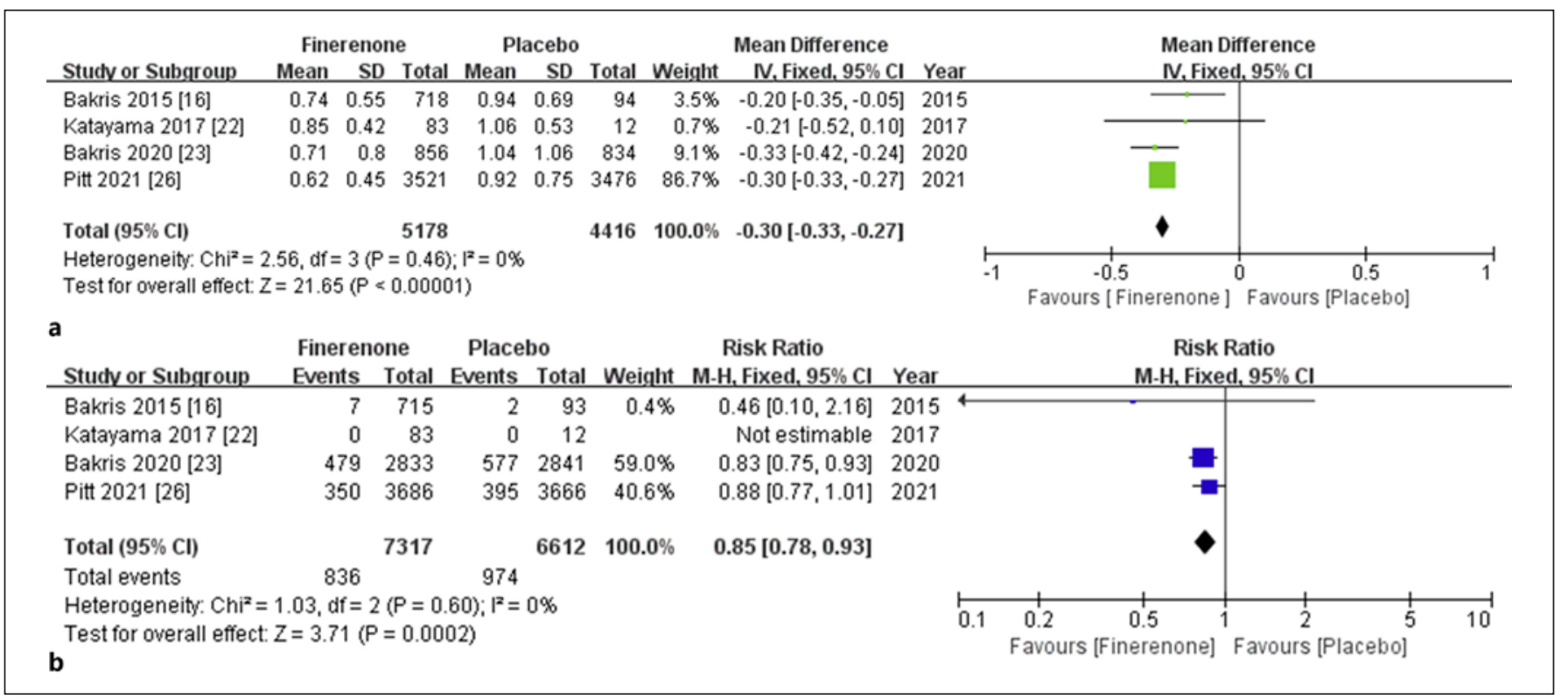

Fig. 3. Comparison between the efficacy of finerenone and placebo on renal function. a Effect on UACR. b Effect on eGFR.

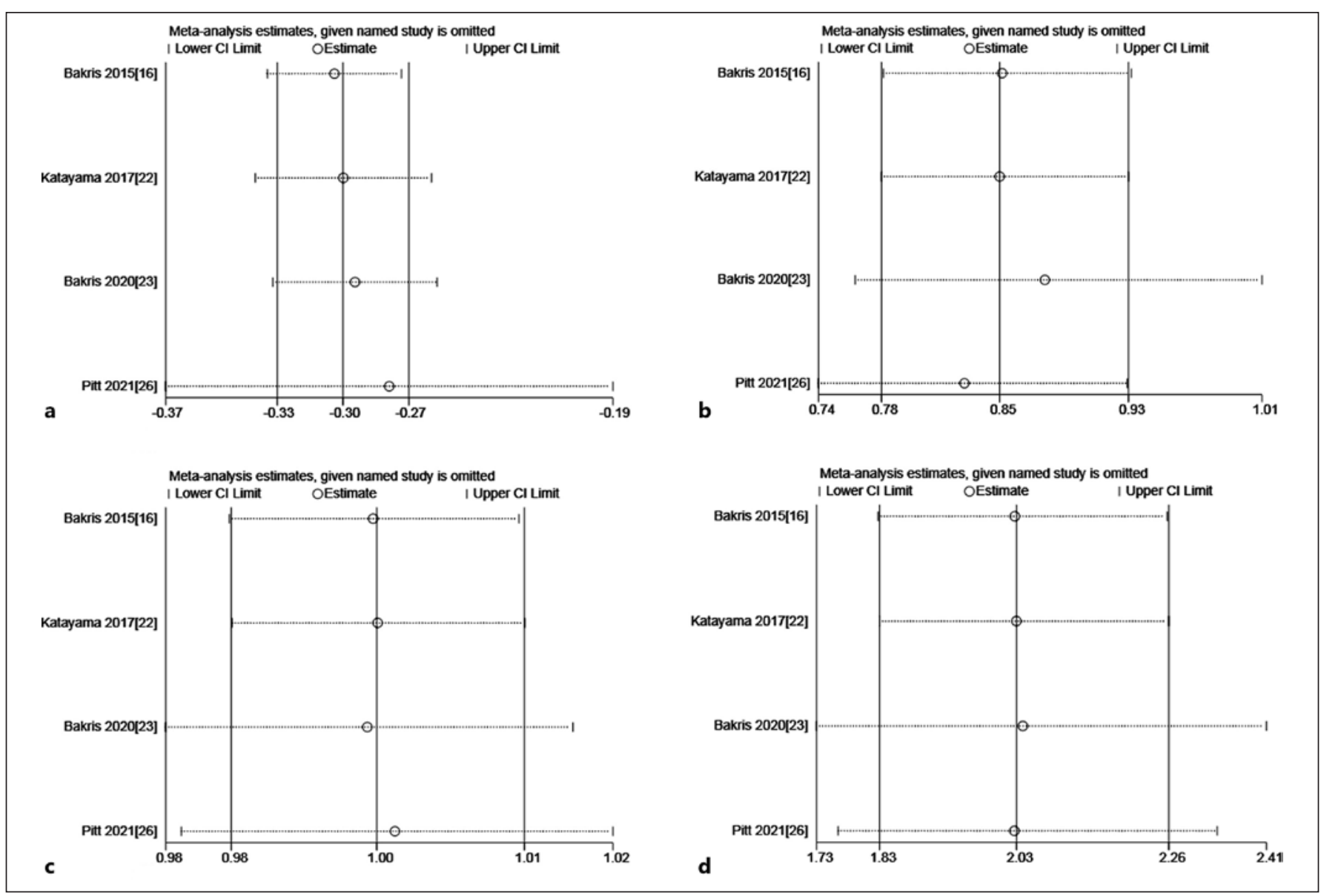

Fig. 4. Sensitivity analysis for interested outcomes in the involved studies. a Changing of UACR from baseline. $\mathbf{b}$ Decrease of $\geq 40 \%$ in eGFR of baseline. c Adverse events. $\mathbf{d}$ Serum potassium $\geq 5.6 \mathrm{mmol} / \mathrm{L}$.

Meta-Analysis of the Efficacy and Safety of Finerenone in DKD
Kidney Blood Press Res 2022;47:219-228

DOI: $10.1159 / 000521908$ 


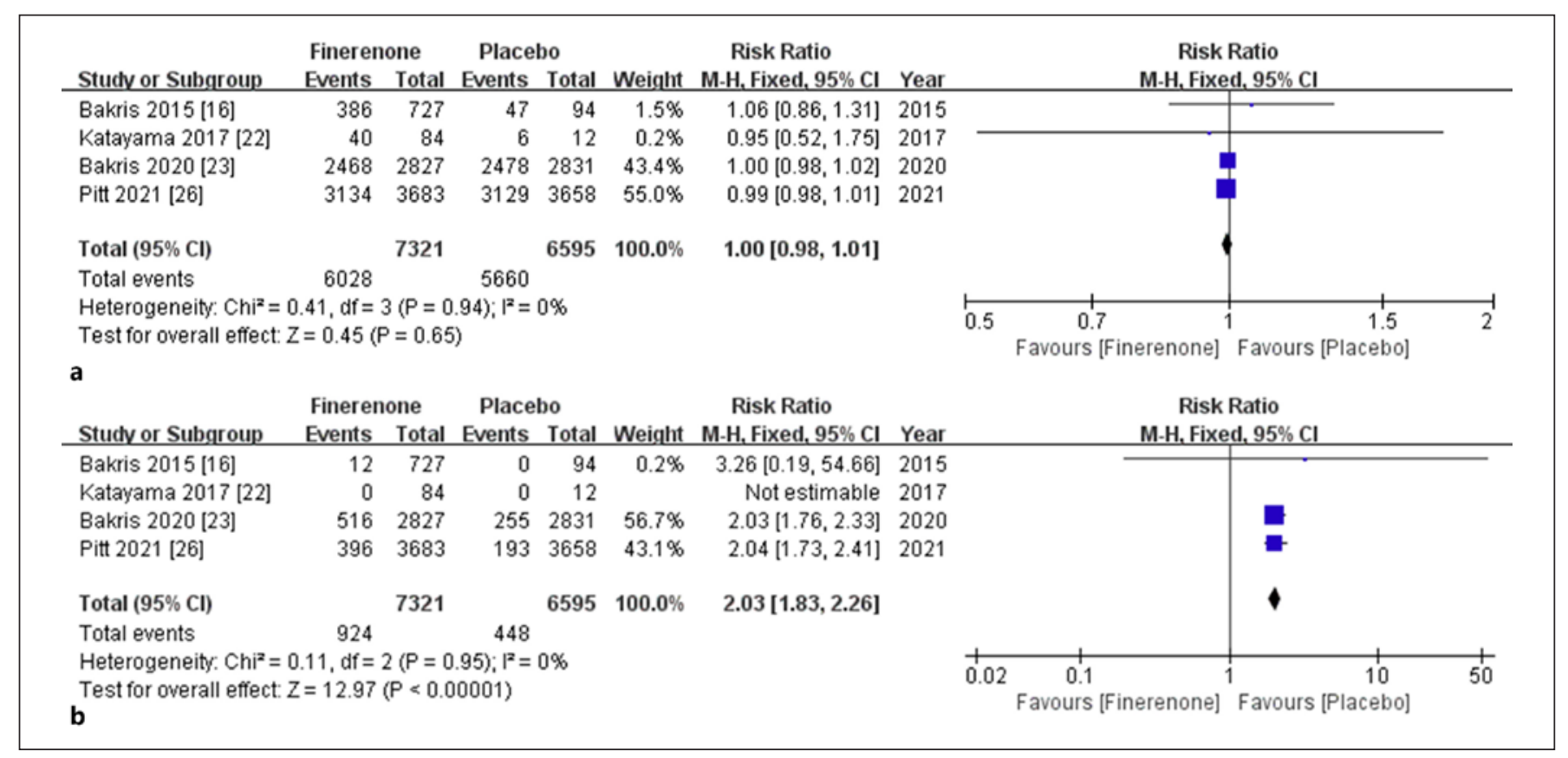

Fig. 5. Comparison between finerenone and placebo in adverse events. a Incidence of any adverse event. b Incidence of hyperkalemia.

was that compared with FIGARO-DKD, the incidence of hyperkalemia was higher in the finerenone group in FIDELIO-DKD. Patients with moderate chronic kidney disease at baseline accounted for $88 \%$ in FIDELIODKD, but $38 \%$ in FIGARO-DKD. Furthermore, there was no restriction on dietary potassium in the clinical trial, ignoring the participants with moderate kidney dysfunction. Hence, for patients with T2D and chronic kidney disease treated with finerenone, attention should be paid to routine monitoring of serum potassium and dietary potassium restriction.

The limitation of this meta-analysis lies in the small number of included studies and the lack of data on some results such as blood pressure. Our study concentrated solely on the effect of finerenone on renal function, but fortunately, a multicenter phase III RCT of FIGARODKD has demonstrated the effect of finerenone on CV in T2D patients with CKD $[26,36]$. In addition, all the four RCTs included in our research were funded by the same company that produced the drug (finerenone), which might bring in financial conflicts of interest and risk of bias in trial result and pooled result in meta-analysis. We look forward to more and more clinical trials focusing on the efficacy and safety of finerenone in the treatment of DKD.

\section{Conclusion}

Finerenone has a beneficial effect on UACR in patients with T2D and chronic kidney disease and can also ameliorate the deterioration of renal function in patients with moderate chronic kidney disease in long-term use. The risk of hyperkalemia was higher in the finerenone group compared with placebo; however, there was no difference in the risk of overall adverse events.

\section{Statement of Ethics}

An ethics statement is not applicable because this study is based on the published literature.

\section{Conflict of Interest Statement}

The authors have no conflicts of interest to declare.

\section{Funding Sources}

This study was supported by the National Health Commission Research Department of Hubei (WJ2021M090), the Nature Science Foundation of Hubei (2020CFB866), Xianning Science and Technology Bureau (2020ZRKX02, 2021ZRKX039), Hubei University of Science and Technology (2021WG03). 


\section{Author Contributions}

Conceptualization and design: Yaning Zheng and Cairong Li. Assessment of studies: Yaning Zheng, Sheng Ma, Cairong Li, and Hongjin Tan. Collection and analysis of data: Yaning Zheng, Sheng Ma, Cairong Li, Qiaomu Huang, and Yu Fang. Writing the article: Yaning Zheng, Sheng Ma, Cairong Li, Qiaomu Huang, Yu Fang, and Hongjin Tan. Supervision: Cairong Li and Yong Chen. Funding acquisition: Cairong Li and Yong Chen.

\section{Data Availability Statement}

All data generated and analyzed during this study are included in this article and its online supplementary material. Details can be consulted from the corresponding author.

\section{References}

1 Garcia-Garcia G, Jha V. CKD in disadvantaged populations. J Nephrol. 2015 Feb;28(2): $1-5$.

2 Xie Y, Bowe B, Mokdad AH, Xian H, Yan Y, Li T, et al. Analysis of the Global Burden of Disease study highlights the global, regional, and national trends of chronic kidney disease epidemiology from 1990 to 2016 . Kidney Int. 2018 Sep;94(3):567-81.

3 Luyckx VA, Cherney DZI, Bello AK. Preventing CKD in developed countries. Kidney Int Rep. 2020 Mar;5(3):263-77.

4 Saeedi P, Petersohn I, Salpea P, Malanda B, Karuranga S, Unwin N, et al. Global and regional diabetes prevalence estimates for 2019 and projections for 2030 and 2045: results from the International Diabetes Federation Diabetes Atlas, 9th edition. Diabetes Res Clin Pract. 2019 Nov; 157:107843.

5 Tuttle KR, Bakris GL, Bilous RW, Chiang JL, de Boer IH, Goldstein-Fuchs J, et al. Diabetic kidney disease: a report from an ADA Consensus Conference. Diabetes Care. 2014 Oct; 37(10):2864-83.

6 de Boer IH, Rue TC, Hall YN, Heagerty PJ, Weiss NS, Himmelfarb J. Temporal trends in the prevalence of diabetic kidney disease in the United States. JAMA. 2011 Jun 22; 305(24):2532-9.

7 Afkarian M, Sachs MC, Kestenbaum B, Hirsch IB, Tuttle KR, Himmelfarb J, et al. Kidney disease and increased mortality risk in type 2 diabetes. J Am Soc Nephrol. 2013 Feb; 24(2):302-8.

8 Wang K, Hu J, Luo T, Wang Y, Yang S, Qing $\mathrm{H}$, et al. Effects of angiotensin-converting enzyme inhibitors and angiotensin II receptor blockers on all-cause mortality and renal outcomes in patients with diabetes and albuminuria: a systematic review and meta-analysis. Kidney Blood Press Res. 2018;43(3):768-79.

9 Association AD. Microvascular complications and foot care: standards of medical care in diabetes-2020. Diabetes Care. 2020 Jan; 43(Suppl 1):S135-51.

10 Fernandez-Fernandez B, Sarafidis P, Kanbay M, Navarro-González JF, Soler MJ, Górriz JL, et al. SGLT2 inhibitors for non-diabetic kidney disease: drugs to treat CKD that also improve glycaemia. Clin Kidney J. 2020 Oct; 13(5):728-33.
11 Wada T, Mori-Anai K, Kawaguchi Y, Katsumata $H$, Tsuda $H$, Iida $M$, et al. Renal, cardiovascular and safety outcomes of canagliflozin in patients with type 2 diabetes and nephropathy in east and southeast Asian countries: results from the CREDENCE Trial. J Diabetes Investig. 2022 Jan;13(1):54-64.

12 Bärfacker L, Kuhl A, Hillisch A, Grosser R, Figueroa-Pérez S, Heckroth H, et al. Discovery of BAY 94-8862: a nonsteroidal antagonist of the mineralocorticoid receptor for the treatment of cardiorenal diseases. ChemMed Chem. 2012 Aug;7(8):1385-403.

13 Rico-Mesa JS, White A, Ahmadian-Tehrani A, Anderson AS. Mineralocorticoid receptor antagonists: a comprehensive review of finerenone. Curr Cardiol Rep. 2020 Sep 10; 22(11): 140 .

14 Pitt B, Kober L, Ponikowski P, Gheorghiade M, Filippatos G, Krum H, et al. Safety and tolerability of the novel non-steroidal mineralocorticoid receptor antagonist BAY 94-8862 in patients with chronic heart failure and mild or moderate chronic kidney disease: a randomized, double-blind trial. Eur Heart J. 2013 Aug;34(31):2453-63.

15 Kolkhof P, Delbeck M, Kretschmer A, Steinke W, Hartmann E, Bärfacker L, et al. Finerenone, a novel selective nonsteroidal mineralocorticoid receptor antagonist protects from rat cardiorenal injury. J Cardiovasc Pharmacol. 2014 Jul;64(1):69-78.

16 Bakris GL, Agarwal R, Chan JC, Cooper ME, Gansevoort RT, Haller H, et al. Effect of finerenone on albuminuria in patients with diabetic nephropathy: a randomized clinical trial. JAMA. 2015 Sep 1;314(9):884-94.

17 Kolkhof P, Jaisser F, Kim SY, Filippatos G, Nowack C, Pitt B. Steroidal and novel nonsteroidal mineralocorticoid receptor antagonists in heart failure and cardiorenal diseases: comparison at bench and bedside. Handb Exp Pharmacol. 2017;243:271-305.

18 Grune J, Beyhoff N, Smeir E, Chudek R, Blumrich A, Ban Z, et al. Selective mineralocorticoid receptor cofactor modulation as molecular basis for finerenone's antifibrotic activity. Hypertension. 2018 Apr;71(4): 599-608.
19 Pei H, Wang W, Zhao D, Wang L, Su GH, Zhao Z. The use of a novel non-steroidal mineralocorticoid receptor antagonist finerenone for the treatment of chronic heart failure: a systematic review and meta-analysis. Medicine. 2018 Apr;97(16):e0254.

20 Fu Z, Geng X, Chi K, Song C, Wu D, Liu C, et al. Efficacy and safety of finerenone in patients with chronic kidney disease: a systematic review with meta-analysis and trial sequential analysis. Ann Palliat Med. 2021 Jul; 10(7):7428-39.

21 Higgins JPT. Cochrane handbook for systematic reviews of interventions version 6.1. John Wiley \& Sons; 2020.

22 Katayama S, Yamada D, Nakayama M, Yamada T, Myoishi M, Kato M, et al. A randomized controlled study of finerenone versus placebo in Japanese patients with type 2 diabetes mellitus and diabetic nephropathy. J Diabetes Complications. 2017 Apr;31(4):758-65.

23 Bakris GL, Agarwal R, Anker SD, Pitt B, Ruilope LM, Rossing P, et al. Effect of finerenone on chronic kidney disease outcomes in type 2 diabetes. N Engl J Med. 2020 Dec 3; 383(23):2219-29.

24 Filippatos G, Anker SD, Agarwal R, Pitt B, Ruilope LM, Rossing P, et al. Finerenone and cardiovascular outcomes in patients with chronic kidney disease and type 2 diabetes. Circulation. 2021 Feb 9;143(6):54052.

25 Filippatos G, Bakris GL, Pitt B, Agarwal R, Rossing P, Ruilope LM, et al. Finerenone reduces new-onset atrial fibrillation in patients with chronic kidney disease and type 2 diabetes. J Am Coll Cardiol. 2021 Jul 13;78(2):14252.

26 Pitt B, Filippatos G, Agarwal R, Anker SD, Bakris GL, Rossing P, et al. Cardiovascular events with finerenone in kidney disease and type 2 diabetes. N Engl J Med. 2021 Dec 9; 385(24):2252-63.

27 Ninomiya T, Perkovic V, de Galan BE, Zoungas S, Pillai A, Jardine M, et al. Albuminuria and kidney function independently predict cardiovascular and renal outcomes in diabetes. J Am Soc Nephrol. 2009 Aug;20(8):181321. 
28 Schmieder RE, Schutte R, Schumacher H, Böhm M, Mancia G, Weber MA, et al. Mortality and morbidity in relation to changes in albuminuria, glucose status and systolic blood pressure: an analysis of the ONTARGET and TRANSCEND studies. Diabetologia. 2014 Oct;57(10):2019-29.

29 Yamout H, Lazich I, Bakris GL. Blood pressure, hypertension, RAAS blockade, and drug therapy in diabetic kidney disease. Adv Chronic Kidney Dis. 2014 May;21(3):281-6.

30 Filippatos G, Anker SD, Böhm M, Gheorghiade M, Køber L, Krum H, et al. A randomized controlled study of finerenone vs. eplerenone in patients with worsening chronic heart failure and diabetes mellitus and/or chronic kidney disease. Eur Heart J. 2016 Jul 14;37(27): 2105-14.

31 Sato N, Ajioka M, Yamada T, Kato M, Myoishi M, Yamada T, et al. A randomized con- trolled study of finerenone vs. eplerenone in Japanese patients with worsening chronic heart failure and diabetes and/or chronic kidney disease. Circ J. 2016 Apr 25;80(5):111322.

32 Agarwal R, Kolkhof P, Bakris G, Bauersachs J, Haller H, Wada T, et al. Steroidal and nonsteroidal mineralocorticoid receptor antagonists in cardiorenal medicine. Eur Heart J. 2021 Jan 7;42(2):152-61.

33 González-Blázquez R, Somoza B, Gil-Ortega M, Martín Ramos M, Ramiro-Cortijo D, Vega-Martín E, et al. Finerenone attenuates endothelial dysfunction and albuminuria in a chronic kidney disease model by a reduction in oxidative stress. Front Pharmacol. 2018;9: 1131.

34 Bauersachs J, Jaisser F, Toto R. Mineralocorticoid receptor activation and mineralocorticoid receptor antagonist treatment in cardiac and renal diseases. Hypertension. 2015 Feb; 65(2):257-63.

35 Bakris GL, Agarwal R, Anker SD, Pitt B, Ruilope LM, Nowack C, et al. Design and baseline characteristics of the finerenone in reducing kidney failure and disease progression in diabetic kidney disease trial. Am J Nephrol. 2019;50(5):333-44.

36 Ruilope LM, Agarwal R, Anker SD, Bakris GL, Filippatos G, Nowack C, et al. Design and baseline characteristics of the finerenone in reducing cardiovascular mortality and morbidity in diabetic kidney disease trial. Am J Nephrol. 2019;50(5):345-56.

37 Pitt B, Pedro Ferreira J, Zannad F. Mineralocorticoid receptor antagonists in patients with heart failure: current experience and future perspectives. Eur Heart J Cardiovasc Pharmacother. 2017 Jan;3(1): $48-57$. 